\title{
Editorial: Advances in Analytical Features of Electrochemical Methods for the Analysis of Complicated Real Samples
}

\author{
Masoud Fouladgar ${ }^{1 *}$, Hassan Karimi-maleh ${ }^{2}$ and Fatih Sen ${ }^{3}$ \\ ${ }^{1}$ Department of Biochemistry, Falavarjan Branch, Islamic Azad University, Isfahan, Iran, ${ }^{2}$ Department of Chemical Engineering, \\ Quchan University of Technology, Quchan, Iran, ${ }^{3}$ Sen Research Group, Biochemistry Department, Faculty of Arts and Science, \\ Dumlupinar University, Evliya Çelebi Campus, Kütahya, Turkey
}

Keywords: electrochemical methods, real sample, complicated matrices, sensors, selectivity

Editorial on the Research Topic

Advances in Analytical Features of Electrochemical Methods for the Analysis of Complicated Real Samples

The aim of this topic was presentation of several features of electrochemical methods for analysis of real samples.

In the last few decades, with the technical progress of electrochemical devices, much attention has been paid to electrochemical methods for analysis of different species. However, like other analytical methods, there has been the challenge of analyzing real samples with complex matrices and several interferences.

Several techniques have been presented to improve selectivity and other figures of merit. Most of them are based on modification of electrodes and creating selective electoactive sites on the surface of electrodes (Fouladgar, 2016). In addition, the modifiers may have electrocatalytic effect on the redox of analyte.

On the other hand, application of nanomaterials has become popular because of increasing the active surface area of electrodes and consequently increasing sensitivity (Negahban et al., 2017).

Papers in this research topic, present various aspects of new methods and techniques, which have been applied for analysis of complicated samples.

As mentioned above, nanomaterials are widely used for modification of electrodes. Metal oxides are among the nanomaterials that used for this purpose. They are applied single or combination with other nanomaterials (Fouladgar and Mohammadzadeh, 2014). Naghian et al. have exhibited that using nanostructures including $\mathrm{CdO}$ and magnetic $\mathrm{Fe}_{3} \mathrm{O}_{4}$ nanoparticles can improve kinetics of the electron transfer process on the electrode. They applied this effect to determine an antiviral drug in urine, plasma and tablet. Moreover, different inherent redox potential of species or different interaction of species with electrode modifier can make simultaneous measurement possible. Accordingly, Pan et al. have reported an electrochemical method for simultaneous determination of Ascorbic acid, dopamine and uric acid in pharmaceutical samples. To reduce utilizing chemical reagents, Jing et al. have used Plectranthus amboinicus leaf extract for biosynthesis of gold nanoparticles. They determined nicotine in tobacco products by modifying glassy carbon with these nanoparticles. 
Feng et al. presented an electrochemical method for determination of ursolic acid in a fruit extract (Ligustrum lucidum) which has many biological effects. Deposited composite of boron nitride nanosheets and $\mathrm{Pt}$ nanoparticles on the surface of glassy carbon, exhibits catalytic activity toward ursolic acid oxidation.

In addition to the analytical aspects of a sensor, biocompatibility of the sensor is important for analysis of clinical samples. Naderi Asrami et al. have prepared a biocompatible electrode containing glucose oxidase enzyme, which was covalently immobilized on a multi-layer thin film electrode. This electrode can be used to direct determination of glucose in blood serum.

Hou et al. presented another aspect of electrochemical methods based on changing piezoelectric properties of quartz crystal by deposition of analyte on it. They immobilized antibodies against B. bifidum on an Au chip and used it as an immunosensor. This kind of sensor has both sensitivity of quartz crystal and specification of immune response between antigen and antibody. Shen et al. have described application of signal amplification technique for creating an immunosensor to determine alpha-fetoprotein in serum samples. They used an immune complex consisting of alpha-fetoprotein antibody and horseradish peroxidase for immune response. In addition, the catalytic effect of a nanocomposite containing graphene oxide, methylene blue and gold nanoparticles has been applied for fabrication of this immunosensor.

One area in which application of electrochemical methods has received much attention is analysis of pharmacological and biological samples. Electrochemistry has presented simple, fast and sensitive methods to analyze these samples. Alizadeh et al.

\section{REFERENCES}

Fouladgar, M. (2016). Application of $\mathrm{ZnO}$ nanoparticle/ion liquid modified carbon paste electrode for determination of isoproterenol in pharmaceutical and biological samples. J. Electrochem. Soc. 163, B38-B42. doi:10.1149/2. 0611603jes

Fouladgar, M., and Mohammadzadeh, S. (2014). Determination of methimazole on a multiwall carbon nanotube titanium dioxide nanoparticle paste electrode. Anal. Lett. 47, 763-777. doi:10.1080/ 00032719.2013 .855782

Negahban, S., Fouladgar, M., and Amiri, G. (2017). Improve the performance of carbon paste electrodes for determination of dobutamine using $\mathrm{MnZnFe} 2 \mathrm{O} 4$ have used effects of modifying electrodes with different nanomaterials and ionic liquid.

Increasing conductivity of carbon paste subsequently increases the redox current and also increases sensitivity of the method. This technique has provided methods for determination of drugs in pharmaceutical samples (Alizadeh et al.).

Remote sensing is important for monitoring chemical species in the environment. Kim et al. have proposed a wireless sensing system for real time measuring chloride ion. They have succeeded in reducing electrolyte leakage by modifying the structure of the electrode.

Finally, the effect of species on the profile of electrochemical oscillation systems has been used for identification chemical compounds. Yan et al. have been shown that recording the oscillation profile of an oscillation reaction in the presence of different herbal medicine can be used as a fingerprint for identification of them.

\section{AUTHOR CONTRIBUTIONS}

MF drafted the manuscript. All co-authors contributed to review the manuscript.

\section{ACKNOWLEDGMENTS}

We want to thank all the authors and reviewers for their valuable contributions to this research topic, and we hope that this collection of articles will be helpful for researchers and students.

nanoparticles and ionic liquid. J. Taiwan Inst. Chem. Eng. 78, 51-55. doi:10. 1016/j.jtice.2017.05.032

Conflict of Interest: The authors declare that the research was conducted in the absence of any commercial or financial relationships that could be construed as a potential conflict of interest.

Copyright (c) 2021 Fouladgar, Karimi-maleh and Sen. This is an open-access article distributed under the terms of the Creative Commons Attribution License (CC BY). The use, distribution or reproduction in other forums is permitted, provided the original author(s) and the copyright owner(s) are credited and that the original publication in this journal is cited, in accordance with accepted academic practice. No use, distribution or reproduction is permitted which does not comply with these terms. 